\title{
Use of Telemedicine in Care of Hematologic Malignancy Patients: Challenges and Opportunities
}

\author{
Jennifer Lloyd ${ }^{1} \mathbb{0}$. Catherine J. Lee ${ }^{1}$ \\ Accepted: 25 May 2021 / Published online: 11 January 2022 \\ (c) The Author(s), under exclusive licence to Springer Science+Business Media, LLC, part of Springer Nature 2021
}

\begin{abstract}
Purpose of Review For nearly 20 years, oncology specialty practices have been working to integrate telemedicine technologies into standard patient care models. However, hematology practices have been slower to adopt telemedicine due to traditional care models that rely on interdisciplinary regional care centers and their ability to provide comprehensive and centralized services. Patients have traditionally been able to access high-quality medical care, diagnostics, supportive care, and clinical trials from these regional care centers, but they are required to attend frequent in-person visits to access these services. Rural and underserved patients experience more barriers than their urban counterparts to access the same level of care. Recent Findings The COVID-19 pandemic has elevated telemedicine into the forefront of care, highlighting both promise and limitations to incorporating telemedicine into specialty hematology care.

Summary Hematologists should consider the benefits of incorporating telemedicine technologies into standard-of-care practices to promote patient-centered care and provide equal access to all patient populations.
\end{abstract}

Keywords Telemedicine $\cdot$ Hematologic malignancy $\cdot$ HCT transplant $\cdot$ COVID-19

\section{Introduction}

Telemedicine, broadly defined here as the use of technology (Internet, telephone/smartphones, or other mobile wireless devices) to provide healthcare over distance, is having increasing importance in the delivery of cancer care. The use of telemedicine in the care of patients with hematologic malignancies is developing. Much of the work providing cancer care through telemedicine has been in the solid tumor setting, forming a framework that hematologists can adapt to clinical practice.

This article is part of the Topical Collection on Health Economics

Jennifer Lloyd

jennifer.lloyd@hci.utah.edu

Catherine J. Lee

catherine.lee@hci.utah.edu

1 Utah Blood and Marrow Transplant Program, Division of Hematology and Hematologic Malignancies, Huntsman Cancer Institute, University of Utah, 2000 Circle of Hope, Clinic 2E, Salt Lake City, UT 84112, USA

\section{History of Telemedicine in Oncology}

The Centers for Medicare \& Medicaid (CMS) implementation of an Electronic Medical Records (EHR) system and promotion of systemic interoperability [1] set the stage for expansion of technology in healthcare. Furthermore, the Health Information Technology for Economic and Clinical Health (HITECH) Act of 2009 secured the establishment of a national initiative to promote healthcare technology tools and established recommendations for the use of technology throughout the continuum of cancer care [2]. These pivotal changes have significantly impacted cancer patients with limited access to care, such as those from rural and frontier areas.

The 2001 National Institute of Health report, Crossing the Quality Chasm: A New Health System for the 21st Century, highlighted the need to develop a healthcare system to overcome quality gaps, transition to evidence-based care practices, and adapt to the explosion of healthcare technologies. They provided recommendations to create a patient-centered approach that incorporates interdisciplinary care teams and also highlighted the need to provide clinical expertise in specialty care in a way that reaches a wide array of patients [3]. These recommendations were still in development when, in 
2012, the Institute of Medicine (IOM) revised and updated their recommendations specific to cancer care. Very little changed with regard to the recommendations; they still emphasized the need for evidence-based practice and clinical trials with results that could be translated directly into patient care, improved accessibility to specialty care, and utilization of technology to provide high-quality cancer care [4]. As cancer patients have a higher risk of hospitalization and infectious complications than the general population, ASCO has also called for a new model of care planning to ensure that all members of the oncology team are able to work together to provide care across the entire continuum of oncology care $[4,5]$.

\section{Benefits of Telemedicine in Hematologic Malignancy}

The National Cancer Institute (NCI) has designated 51 comprehensive cancer centers, most located next to university medical centers, to centralize specialty care, focus on basic and clinical research, have large transdisciplinary teams, and act as community hubs for cancer care in these regions [6]. These NCI designated centers offer exceptional care with access to cutting-edge treatments but are they truly patientcentered? The patient must travel to the cancer center to receive care and to access large clinical trials of novel therapies that are rarely offered in community clinics. Patients from rural settings or poor socioeconomic backround benefit least from this model as they often do not have the resources to travel long distances and secure lodging and food needed for a visit. According to the American Society of Clinical Oncology (ASCO), these cancer patients have a higher incidence of death as compared to their urban counterparts[7] due to poor access to trained medical oncologists who can provide expert care and cancer clinical trials [8]. Utilizing telemedicine has the potential to meet NCIs mission of supporting patient care and research endeavors that improves the lives of all cancer patients.

Allogenic hematopoietic stem cell transplant (HCT) is a potentially curative treatment for many hematologic malignancies such as leukemias and lymphomas and non-malignant disorders like aplastic anemia [9]. However, patients who receive HCT or who further develop graft-versus-host disease (GVHD) face the highest risk for infections due to prolonged chemotherapy-induced neutropenia, post-transplant pharmacologic immunosuppression, and impaired immune function [10]. A Center for International Blood and Marrow Research study showed recipients of autologous and allogeneic HCT who developed COVID-19 infection to have poor overall survival $[10,11]$. The use of telemedicine for post-HCT care has the potential to minimize infection risk in high-risk patients. For patients entering long-term follow-up post-HCT, telemedicine can help alleviate outof-pocket expenses for travel and lodging, and furthermore reduce missed work [12] which is often a crucial issue for post-HCT patients facing financial hardships.

A review of the literature presented by Sabe Sebesan [13] in the Asian-Pacific Journal of Clinical Oncology highlighted that overall, patients had a high level of satisfaction with video-based telemedicine visits. This included both video visits that were performed from patients' homes as well as those based in local healthcare centers, separate from the specialty oncology center. This is confirmed by more recent research regarding patient satisfaction with video visits performed during COVID-19 pandemic. By analyzing Press Ganey scores, a nationally accepted means of measuring patient satisfaction with clinicians and care systems, the researchers determined that scores were higher for visits performed using video technology than those performed in person [14•]. These may be influenced by patients' fear of leaving their homes or being exposed to infection at clinical settings. Thus, they may be more willing to look favorably on telemedicine as an option for seeking care when their perception of the benefits outweigh risks they perceive to be associated with attending in-person appointments. It does imply that video visits are a viable option in patients' minds as an alternate form to in-person care.

\section{COVID-19 Pandemic}

The COVID-19 pandemic caused by the SARS-Cov2 virus has driven telemedicine into the forefront of healthcare delivery as all care settings have been forced to rapidly adapt from traditional in-person visits to virtual encounters. Not so commonly used in the past for hematology patients, this new model of healthcare delivery has been especially important to patients with hematologic cancers as they are a high-risk category for severe COVID-19 infection and death due to prolonged cytopenias caused by their blood cancer, immunocompromised state and need for active anticancer treatment $[11,15]$. Initial studies have suggested that patients with hematologic disease who develop symptomatic COVID-19 infections have poorer prognosis than those without hematologic disease [16]. Hematology patients attempting to access care during the pandemic not only face the risks of contracting SARS-Cov2 but also face limitations in access to inpatient chemotherapy treatments and acute care services due to medical care rationing caused by the pandemic [17]. The experience detailed by the Hematology and Medical Oncology department of Ney York-Presbeterian shows a significantly increase utilization of telemedicine during the early phases of the pandemic. There was also a decrease in onsite clinical visits, but it was unequal to the increase seen in telemedicine visits [15]. Through telemedicine and 
coordination with local resources, such as laboratory and community oncology settings, hematology patients were able to still access needed care.

\section{Patient-Centered vs. Center-Based Care Models}

The standard paradigm for care of patients with hematologic malignancies is primarily in-person treatment visits at a regional cancer center. They have access to cutting-edge research-based cancer care; however, bringing patients that are vulnerable to infections into the hospital needs reassessment in the face of current care challenges.

Improving access through the use of telemedicine has the potential to bring specialty cancer care directly to the patient's home. For patients with limited accessibility to a comprehensive cancer center, community-based agreements should be developed so that patients can have their cancer care needs managed by a community oncology provider and concurrently have access to NCI specialists for expert opinions through synchronous or asynchronous live video visits in the local clinic or in the patient's home. Models of care that incorporate telemedicine may benefit a diversity of underserved and vulnerable populations, such as adolescent and young adults, elderly or those with highly morbid illnesses, poor socioeconomic status, or rural backgrounds.

High-risk hematology patients receiving chemotherapy, immunosuppressive therapy, or post-transplant care, would also benefit from having telemedicine incorporated into their usual care model. Patients would continue to have close monitoring by providers and clinical staff without the added risk of exposure to illness in the clinic or hospital setting. These visits could be augmented using home health nursing staff for laboratory monitoring, vital sign evaluation, and physical exams. Through the use of cooperative agreements with local healthcare facilities, patients could have access to point-of-care services, including diagnostics, but still have real-time feedback from their hematology team.

\section{Challenges of Telemedicine}

The effectiveness of telemedicine is limited by the technology used for the service. For visits requiring video, patients will need access to devices that have the software platforms necessary to use video technology. They will also need to live in an area with adequate bandwidth and connectivity to support its use. As telemedicine expands to cover more patient populations, this will increase use and potentially overload rural internet and telecommunications infrastructure that were not designed to support a heavy use. Local conditions, such as inclement weather, may also interfere with video connectivity and consequently interfere with communication and understanding between the patient and provider. Inability to receive care recommendations in a timely manner could adversely affect health outcomes.

In a research letter presented in JAMA Internal Medicine [18] as many as $38 \%$ of older adults (those greater than 65 years of age) not living in residential or healthcare facilities have some level of technologic unreadiness. This is a necessary skill to be able to successfully navigate developing telemedicine care platforms. Since hematologic malignancies are primarily a disease of older adults, they have a particular risk of being unprepared to adapt and transition to the growing telemedicine platforms being offered by specialty care providers. For patients who received HCT, lack of Internet access has been shown to cause increased distress after transplant [19]. Without reliable Internet access, patients will be unable to participate in growing telehealth technologies and they may face the possibility of having limited access to care, thereby negatively affecting health and quality of life.

Although video allows providers to perform a limited physical exam, lighting, connection issues, and video quality can affect the usefulness of the exam. Current technology is limited in how in-depth the physical exam can be performed. The clinician is unable to auscultate for lung and cardiac sounds, cannot palpate, and is dependent on the patient to describe physical changes accurately. Fortunately, advancements in technology have also expanded to at-home health monitoring opportunities. Smartphones and watches offer cardiac monitor software, and digital stethoscopes and remote exam technologies could allow patients to use medical devices at home and providers to interpret the data from a distance. However, high costs of these mobile devices can be prohibitive to the use of smart health technologies but could be improved if insurance companies covered home health devices and telehealth systems.

Reimbursement for telemedicine services is a concern for specialty care facilities. Regional cancer care centers not only offer complex medical care, but a variety of interdisciplinary teams, such as nutritional care, physical and occupational therapy, social work and other patient support services, would not only need to adapt their care models to provide distance-based care but may be affected by limitations in billing and reimbursement. Clinicians may also encounter limitations using telemedicine across state lines as licensing is required for each state. Furthermore, reimbursement of providers for telemedicine visits is critical and remains an obstacle for universal implementation of a successful telemedicine program.

Distance from the regional cancer center to the patient does not make the medical need less complex. It potentially complicates medical decisions as access to hospital-based treatments and diagnostic testing may not be available in 
the area where the patient lives. Patients with hematologic malignancies often need hospital services such as inpatient and ICU beds, blood banking services, and utilization of staff that may otherwise be facing shortages due to increased patient volume brought on by the pandemic [20]. Ensuring that the proper infrastructure is in place for patients to be managed safely at home through telemedicine will be important in maintaining current care standards.

Due to the COVID-19 pandemic, CMS decreased organizational financial barriers by altering its medical reimbursement scale to make telemedicine reimbursement equal to in-person visits. This also included audio-only visits for patients who do not have the technology to connect to live video [21]. Many individual states also waived state licensure requirements for telehealth and expedited licensure approval for clinical providers to provide telehealth visits across state lines due to the COVID-19 public health emergency [22]. It remains to be determined how these scales will be adjusted after the current crisis is over and local travel restrictions no longer limit clinical visits. With expanded use of telemedicine, multi-state licensing compacts for providers could improve access by minimizing cross-state barriers and licensing cost.

\section{Opportunities}

Patient access to reliable internet, phone, and technologic devices are limiting factors in the expansion of telemedicine into rural and underserved communities. To facilitate the continued development, it is necessary for local, state, and national governments to invest in the infrastructure needed to provide reliable connectivity across communities. Tools such as the Federal Communication Commissions (FCC) Mapping Broadband Health in America platform helps public entities identify connectivity gaps and explore how they relate to health statistics such as obesity, hospitalizations, and diabetes [23]. Utilizing these tools can allow local governments and healthcare entities to identify areas in the highest need for connectivity improvement. NCI Comprehensive care centers associated with large academic teaching hospitals have an opportunity to address care gaps to support patient technology access. Local community engagement is necessary to ensure that communities will utilize technologic infrastructure once developed.

The dramatic shift in care delivery due to the COVID19 pandemic has opened the doors for multiple research opportunities. Further work needs to be done in the areas of cost effectiveness of ongoing telemedicine use; patient utilization and telemedicine's impact on long-term patient outcomes; utility of patient remote monitoring devices to facilitate home-based care models for HCT and other cellular therapies. CMS emergency measures should be analyzed to see if long-term reimbursement and licensing incentives can encourage continued growth of telemedicine.

\section{Conclusion}

The development of telemedicine in the care of hematology patients is developing, but there are gaps in care due to patient access, disease complexity, and medical acuity. The COVID-19 pandemic has driven telehealth into implementation without a fully developed infrastructure to accommodate the change. Some patients are being left behind due to limited access to and difficulties with technology, and at times, mistrust in the safety of the system. Care models to integrate telehealth technology more fully is necessary to improve care and to continue adapting to changing care environments.

As seen in Table 1, telemedicine is a promising modality for delivering care safely to patients who would otherwise be at risk of infection. Telemedicine does not replace current care models but should be used as an adjunct to improve patient safety, decrease healthcare costs, and reach rural and underserved patients that currently have difficulty accessing

Table 1 Telemedicine use for patients with hematologic malignancies

\begin{tabular}{ll}
\hline Benefits & Challenges \\
\hline - Improve access to rural and underserved populations & • Lack of telecommunication infrastructure in some rural communities \\
- Minimize infectious exposure to high-risk patients with hematologic & • Limited physical exam capabilities \\
malignancies & - Lack of standardized clinical reimbursement \\
- Expand access to clinical expertise and clinical trials & $\bullet$ Need for software training by providers and patients \\
- Maintain clinic relationships with patients over distance & - Need for information technology support for patients and organiza- \\
- Minimize out-of-pocket expenses for patients & tions \\
Opportunities & \\
- Expand relationships with community oncologists and NCI hematologists \\
- Monitoring, and early intervention, treatment, and post-treatment care from the patients home \\
- Work with local healthcare organizations and governments to develop and improve infrastructure \\
- Encourage insurance coverage of healthcare technology for patient access \\
- Expand research opportunities on patient and organizational outcomes
\end{tabular}


regionalized cancer centers. A critical analysis of existing models of care is necessary to protect vulnerable hematology patients as well as improve access to high-quality care. Expanding access to clinical trials will require organizational and healthcare provider buy-in. Centralized research staff can interact with distant patients through telemedicine, and the use of existing EMR and data monitoring technology will ensure the safety of the data.

Nineteen years after the initial IOM report, the care of patients with hematologic malignancies still falls short of the goal of providing truly patient-centered care that is affordable, accessible, and tailored to the patient's individual needs. The COVID-19 pandemic has shown the promise of telemedicine technologies for the care of complex and medically at-risk patients. While strides have been made integrating telemedicine into general oncology care, hematology practices need to develop a multifaceted telemedicine platform to offer high-quality medical care at a distance to patients anywhere in the treatment continuum.

\section{Declarations}

Conflict of Interest The authors declare no competing interests.

Human and Animal Rights and Informed Consent This article does not contain any studies with human or animal subjects performed by any of the authors.

\section{References}

Papers of particular interest, published recently, have been highlighted as:

\section{- Of importance}

1. Lammers EJ, Mclaughlin CG. Meaningful use of electronic health records and medicare expenditures: evidence from a panel data analysis of U.S. health care markets, 2010-2013. Health Serv Res. 2017;52(4):1364-86. https://doi.org/10.1111/14756773.12550.

2. ONC. Laws, regulation, and policy. In: Heath Information Technology. US Department of Health and Human Services, Washington, DC. 2019. https://www.healthit.gov/topic/laws-regul ation-and-policy. Accessed 23 Nov 2020.

3. IOM. Crossing the quality chasm: a new health system for the 21 st century. Washington, DC: National Academies Press (US) 2001.

4. Ganz PA. Institute of Medicine Report on delivery of high-quality cancer care. Journal of Oncology Practice. 2014;10(3):193-5. https://doi.org/10.1200/JOP.2013.001369.

5. Patt DA, Wilfong L, Toth S, Broussard S, Kanipe K, Hammonds $\mathrm{J}$, et al. Telemedicine in community cancer care: how technology helps patients with cancer navigate a pandemic. JCO Oncol Pract. 2021;17(1):e11-5. https://doi.org/10.1200/op.20.00815.
6. NCI. NCI-Designated Cancer Centers. National Cancer Institute. 2019. https://www.cancer.gov/research/infrastructure/cancercenters. Accessed 23 Nov 2020.

7. Blake KD, Moss JL, Gaysynsky A, Srinivasan S, Croyle RT. Making the case for investment in rural cancer control: an analysis of rural cancer incidence, mortality, and funding trends. Cancer Epidemiol Biomark Prev. 2017;26(7):992-7. https://doi.org/10.1158/1055-9965.epi-17-0092.

8. Levit LA, Byatt L, Lyss AP, Paskett ED, Levit K, Kirkwood $\mathrm{K}$, et al. Closing the rural cancer care gap: three institutional approaches. JCO Oncology Practice. 2020;16(7):422-30. https://doi.org/10.1200/OP.20.00174.

9. NMDP. Disease specific HCT indication and outcome data. National Marrow Donor Program. 2021. https://bethematch clinical.org/about-us/trademark-and-copyright/. Accessed 23 Nov 2020.

10. Sahin U, Toprak SK, Atilla PA, Atilla E, Demirer T. An overview of infectious complications after allogeneic hematopoietic stem cell transplantation. J Infect Chemother. 2016;22(8):505-14. https://doi.org/10.1016/j.jiac.2016.05. 006.

11. Sharma A, Bhatt NS, St Martin A, Abid MB, Bloomquist J, Chemaly RF, et al. Clinical characteristics and outcomes of COVID19 in haematopoietic stem-cell transplantation recipients: an observational cohort study. Lancet Haematol. 2021;8(3):e18593. https://doi.org/10.1016/S2352-3026(20)30429-4.

12. Khera N, Martin P, Edsall K, Bonagura A, Burns LJ, Juckett M, et al. Patient-centered care coordination in hematopoietic cell transplantation. Blood Adv. 2017;1(19):1617-27 https://doi.org/ 10.1182/bloodadvances.2017008789.

13. Sabesan S. Medical models of teleoncology: current status and future directions: medical models of teleoncology. Asia Pac J Clin Oncol. 2014;10(3):200-4. https://doi.org/10.1111/ajco. 12225 .

14. - Ramaswamy A, Yu M, Drangsholt S, Ng E, Culligan PJ, Schlegel PN et al. Patient satisfaction with telemedicine during the COVID-19 pandemic: retrospective cohort study. J Med Internet Res. 2020;22(9):e20786-e. doi:https://doi.org/10.2196/ 20786. These papers represent recent work that highlight key aspects addressed in this article regarding telemedicine, clinical impact of COVID-19 on patients with hematopoietic malignancies, and rural health discrepancies in cancer care.

15. Shah MA, Emlen MF, Shore T, Mayer S, Leonard JP, Rossi $\mathrm{A}$, et al. Hematology and oncology clinical care during the coronavirus disease 2019 pandemic. CA Cancer J Clin. 2020;70(5):349-54. https://doi.org/10.3322/caac.21627.

16. Cattaneo C, Daffini R, Pagani C, Salvetti M, Mancini V, Borlenghi $\mathrm{E}$, et al. Clinical characteristics and risk factors for mortality in hematologic patients affected by COVID-19. Cancer. 2020;126(23):5069-76. https://doi.org/10.1002/cncr.33160.

17. Ngo A, Masel D, Cahill C, Blumberg N, Refaai MA. Blood banking and transfusion medicine challenges during the COVID19 pandemic. Clin Lab Med. 2020;40(4):587-601. https://doi. org/10.1016/j.cll.2020.08.013.

18. Lam K, Lu AD, Shi Y, Covinsky KE. Assessing telemedicine unreadiness among older adults in the United States during the COVID-19 pandemic. JAMA Intern Med. 2020;180(10):138991. https://doi.org/10.1001/jamainternmed.2020.2671.

19. Banerjee R, Yi JC, Majhail NS, Jim HSL, Uberti J, Whalen $\mathrm{V}$, et al. Driving distance and patient-reported outcomes in hematopoietic cell transplantation survivors. Biol Blood Marrow Transplant. 2020;26(11):2132-8. https://doi.org/10.1016/j. bbmt.2020.08.002.

20. Raza A, Assal A, Ali AM, Jurcic JG. Rewriting the rules for care of MDS and AML patients in the time of COVID-19. Leuk 
Res Rep. 2020;13: 100201. https://doi.org/10.1016/j.lrr.2020. 100201.

21. CMS. Telemedicine. US Centers for Medicare \& Medicaid Services, Baltimore, MD. 2020. https://www.cms.gov/Medic are/Medicare-General-Information/Telehealth. Accessed 9 Dec 2020.

22. FSMB. U.S. states and territories modifying requirements for telehealth in response to COVID-19. Federation of State Medical Boards; 2021.
23. FCC. Mapping broadband health in America. Federal Communications Commission, Washington DC. 2017. https://www.fcc. gov/health/maps. Accessed 1 Feb 2021.

Publisher's Note Springer Nature remains neutral with regard to jurisdictional claims in published maps and institutional affiliations. 